\title{
A Regularization Blind Image Restoration Technique by Using Particle Swarm Optimization
}

\author{
Lei Xuanhua ${ }^{1}$, Hu Qingping, Kong Xiaojian, Xiong Tianlin
}

\begin{abstract}
Image degradation in laser underwater imaging system is due to the scattering of water. Image restoration is an effective method to improve the quality of image, but its solving process is an ill-posed problem and the solution is not easily obtained in actual applications. A regularization blind image restoration method using particle swarm optimization was proposed in this paper. Regularization technique was adopted to solve ill-posed problem in the process of blind image restoration, the selection of regularization parameter still remains a difficult problem due to the amplification of noise in the inversion process. So two kind of particle swarm optimization were used to estimate regularization parameter and point spread function alternately. The results of image simulation and experiment show that the algorithm has a good effect of restoration for underwater image.
\end{abstract}

Keywords: Blind Image Restoration · Regularization · Particle Swarm Optimization

\section{Introduction}

The main cause of image quality degradation acquired by underwater laser imaging system is the absorption and scattering during laser transmission. Underwater

${ }^{1}$ Lei Xuanhua $(\bowtie)$

Naval Univ.of Engineering, Wuhan 430033, Hubei, China

e-mail: leixuanhua@sina.com 
absorption of water leads to the decrease of image signal noise ratio, scattering leads to blur images. Image inverse filtering is a common way to restore degraded images [1], but knowledge of point spread function must be known. Currently, most of the solution of water point spread function is approximate solution [2], which is related to the intrinsic properties of water (absorption coefficient and scattering coefficient) and imaging distance. So, the solution is not easily obtained in actual applications. Blind image restoration method is a method of processing without the need of a priori knowledge of image, but its solving process is an ill-posed problem [3].

Based on blind image restoration method, a regularization blind image restoration method by using particle swarm optimization is proposed. In this method, regularization technology is adopted to solve ill-posed problems in blind image restoration algorithm, and then particle swarm optimization algorithm is adopted to estimate regularization parameter and point spread function.

\section{Algorithm model and description}

\subsection{Regularization blind image restoration model}

The key of image restoration processing is to establish degradation model. In most of the applications, it is assumed that the image degradation model is linear and can be modeled as a two dimensional convolution between the original image with the point spread function as given [4], In matrix form the observed image can be written as

$g=H f+h$

In formula (1), $\mathrm{g}$ is a degraded image, $H$ point spread function, i.e. water point spread function, $\mathrm{f}$ is original image, and $\mathrm{n}$ represents the additive Gaussian noise.

Because of the diverse factors and character caused image degradation, image restoration is a complex process of mathematical, essentially is the process of solve the ill-posed problem. Regularization method is a kind of good method for solving ill-posed problems $[5,6]$. The basic idea of this method is to make use of prior knowledge of the solution, and to format additional constraints in order to ensure the 
only existence continuous solution, thus to transform the ill-posed problem into well-posed problem. Therefore, regularized image restoration could be regarded as a constraint optimization problem, namely, the optimal value of original image $f$ is:

$f^{\prime}=\arg \min \left\{\|H f-g\|^{2}+\mu\|c(f)\|^{2}\right\}$

In formula (2), $\|H f-g\|^{2}$ represents the approximation degree of observation image to original image, $\mu$ is the regularization parameter, $c$ represents the regularization operator, usually it is a high-pass filter operator so as to realize noise smoothing, $\|c(f)\|^{2}$ represents regular term and $f^{\prime}$ is for regular solution[7].

For a given regularization operator $c$, we could select proper solution regularization parameter $\mu$, and then by calculating with formula (3), regularization solution $f^{\prime}$ could be obtained:

$$
f^{\prime}(\mu)=\left(H^{T} H+\mu c^{T} c\right)^{-1} H^{T} g
$$

For $\mu$ optimal value, mean square error is:

$$
e(\mu)=\frac{1}{N}\|H f(\mu)-g\|^{2}
$$

Various methods for regularization parameter were used to solve this problem [8-10], but how to choose regularization parameters is a challenge problem yet, and the parameters very close to optimum recovery are rare. In order to get the optimal solution, alternating optimization is a kind of effective method. In this way, image blind restoration process is divided into fuzzy identification and regularization parameter optimization this two alternating stage. In fuzzy identification stage, solve $H$ by fixing regularization parameter $\mu$ and minimizing the optimization performance index in formula (2). In regularization optimization stage, solve $\mu$ by fixing $H$ and minimizing the optimization performance index in formula (4).

The algorithm model is as follows:

Step1: Initialize $\mu, H, c$ is laplacian operator

Step2: Calculate $f^{\prime}$ using equation (3)

Step3: Fuzzy identification (PSO1), using equation (2) 
Step4: Optimize the regularization parameter (PSO2) using equation (4)

Step5: Number of iterations is satisfied, if not satisfied to Step3

Step6: output $f^{\prime}$.

\subsection{PSO algorithm}

Particle swarm optimization algorithm is a new global optimization evolutionary algorithm, which is proposed by Kennedy and Eberhart in 1995 [12] and is especially suitable for nonlinear function optimization problem. In this algorithm, firstly, a group of particles are generates randomly in the solution space and then the optimal solution is found through iteration. During every time of iteration, particle updates itself by tracking two "extreme value". Suppose that one is the best solution the particle itself finds, called $p_{\text {best }}$, the other one is the best solution the whole particle swarm could find at present, called $g_{\text {best }}$. Hence, update equation of the particle's speed and location [13] are:

$V_{\text {step }}=w_{\text {now }} * V_{\text {step }}+c_{1} *$ rand $*\left(p_{\text {best }}-s_{\text {warm }}\right)+c_{2} *$ rand $*\left(g_{\text {best }}-s_{\text {warm }}\right)$

$s_{\text {warm }}=s_{\text {warm }}+V_{\text {step }}$

In formula (5), $V_{\text {step }}$ is the speed of particle, $S_{\text {warm }}$ is the current position of particle, rand is respectively the random number between $[0,1], c_{1}, c_{2}$ is learning factor,

$w_{n o w}$ is inertial factor, usually linearly reduces with the increase of iterations, i.e.

$w_{\text {now }}=w_{\text {now }}-$ Iteration $* \frac{w_{\text {start }}-w_{\text {end }}}{\text { IterationMax }}$

$w_{\text {now }}$ Is biggest inertial factor, generally take value $0.9, w_{\text {end }}$ is the minimum inertia 
factor, general take value 0.1, Iteration is the number of iterations, IterationMax is the maximum number of iterations. In this paper the size of particle swarm is set as 20. PSO pseudo-code is as follows:

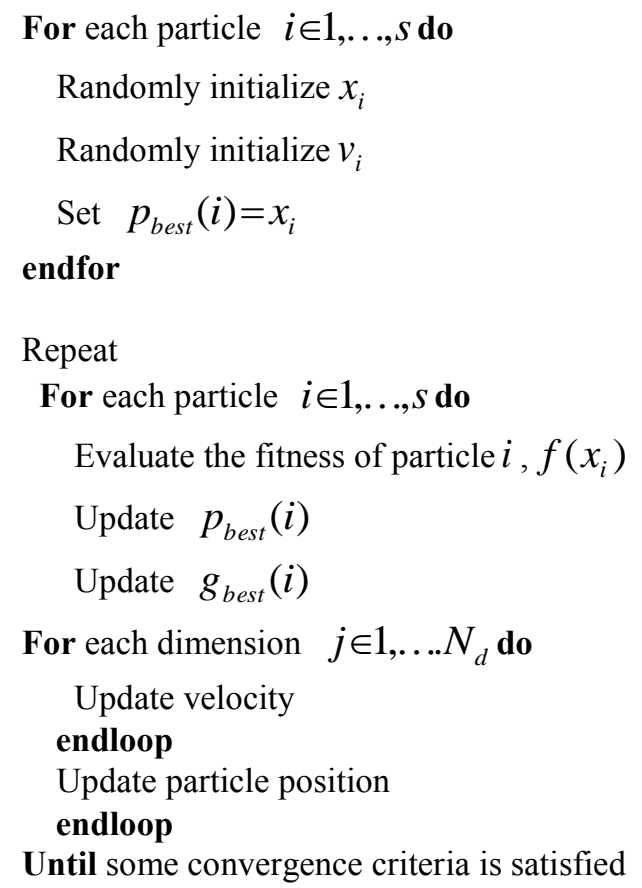

\section{Simulation experiment and analysis of results}

In order to verify performance of the algorithm proposed in this paper, simulation image and actual underwater image were restored separately by algorithm proposed in this paper and the other two kinds of algorithms proposed in literature [14] [15]. Then, we compared the image restoration result of each algorithms and adopted signal-to-noise ratio increment ISNR to objectively measure the performance of each blind restoration method [16].

\subsection{Comparison of the restoration algorithm for image simulation}

The original picture Lena bellow is chosen from the standard image base, By 
Gaussian Blur and adding certain amount of noise, results are shown in figure 1.

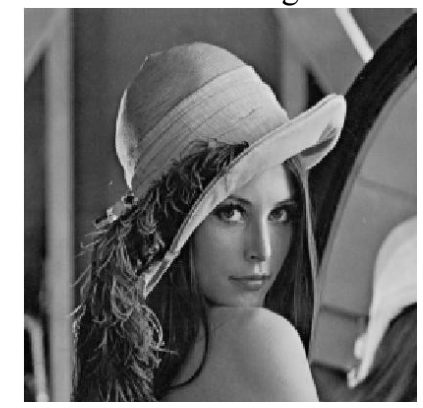

(a) Original image Lena

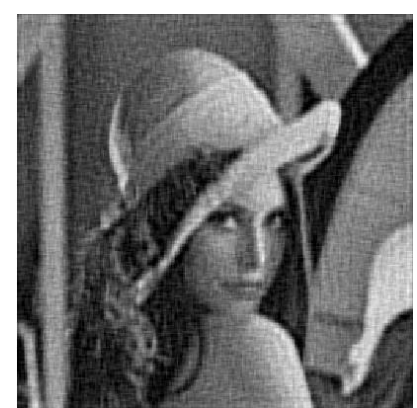

(c) result of method in literature [15]

$\mathrm{ISNR}=1.8 \mathrm{db}$

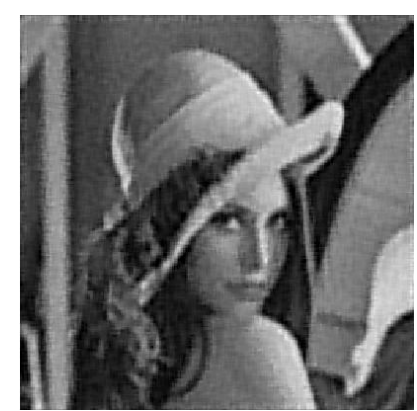

(e) Result of method in this paper

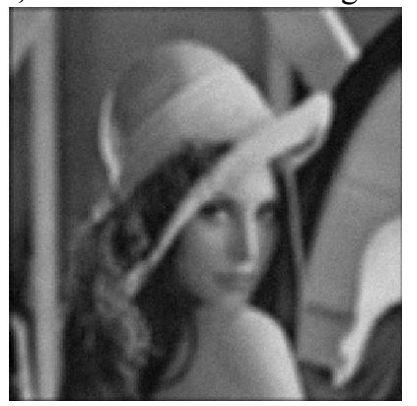

(b) Gaussian blur image quality image

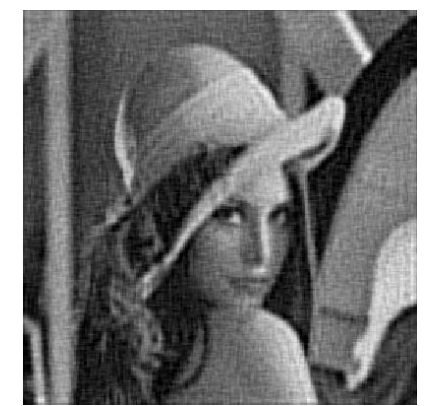

(d) result of method in literature [14] ISNR $=2.1 \mathrm{db}$ $\mathrm{ISNR}=3.2 \mathrm{db}$

Fig. 1 Recovery results of different blind restoration algorithm for the simulation diagram

From recovery effect, restoration images obtained by dealing with algorithm proposed in this paper and the other two kinds of algorithms are all clean and clear, 
Contrast and detail have been obviously improved and a good visual effect is got. But, restoration images obtained by dealing with the algorithm proposed in literature [14] [15] have different degree of blocking effects, restoration images obtained by dealing with algorithm proposed in this paper has a better effect.

\subsection{Blind restoration for real image}

Real blur image obtained by the laser underwater imaging system are selected in this paper. As shown in figure 2(a). Then blind restoration is preceded by the algorithm proposed in literature [14] and [5] and this article. Recovery effect is shown in figure 2(b), 2(c), 2(d) respectively.
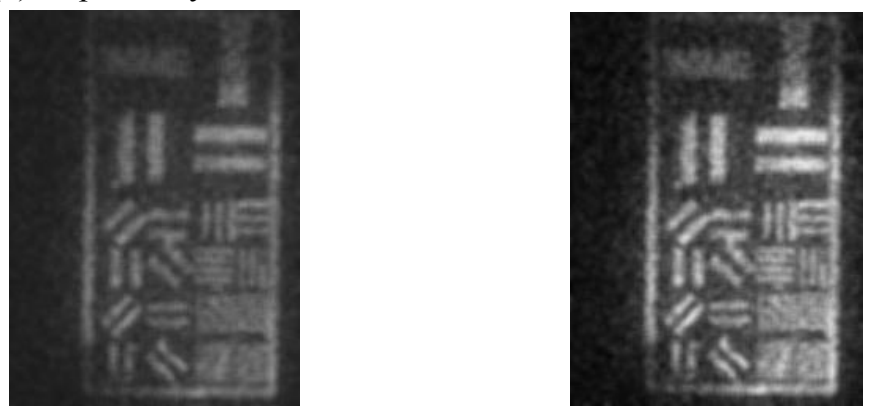

(a) Real underwater image

(b) result of literature [15] method
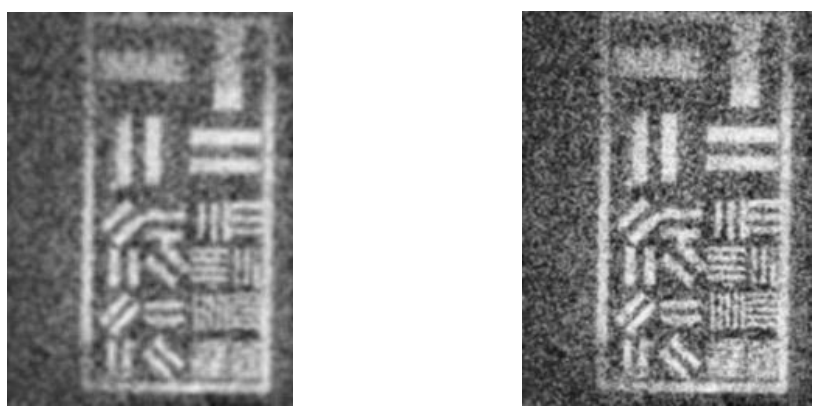

(c) result of literature [14] method

(d) result of this paper method

Fig. 2 Recovery results of different blind restoration algorithm for the simulation diagram

As shown in the figure 2, certain degree of recovery for image restoration are obtained by this three restoration algorithms, but effects of detail and texture on the 
recovery image obtained by the algorithm proposed in this paper is more prominent than other two. Because the original clear image that could not be obtained, so only subjective judgment could be made by us without reference. Blind restoration for real blur image has been well realized by the algorithm proposed in this article.

\section{Conclusions}

Image degradation in laser underwater imaging system is due to the scattering of water. It is a very challenging problem for image blind restoration under the environment of lack of prior knowledge of the image. Particle swarm optimization algorithm proposed in this paper for regular blind image restoration technology can effectively solve the ill-posed problem of blind restoration. The results of image simulation and experiment show that the algorithm has a better effect in estimating regular parameters and original image.

\section{References}

1. Bonham M. R., Katsaggelos A. K. (1997). Digital Image Restoration. IEEE Signal Processing Magazine, 14(2):24-41.

2. Sanchez R., McCormick N.J. (2002). Analytic beam spread function for ocean optics applications. Applied Optics, 41(30):6276-6288.

3. Kundur D., Hatzinakos D. (1996). Blind Image Deconvolution. IEEE Signal Processing Magazine, 43-64.

4. Gonzalez R.C., Woods R.E. (1992). Digital Image Processing. 2nd Addison Wesley.

5. Katsaggelos A.K.(1991). Digital Image Restoration. Berlin: Springer-Verlag.

6. Andrews H., Hunt B. (1977). Digital Image Restoration. Englewood Cliff, NJ: Prentice-Hall.

7. Barter M., Poggio T., Torre V. (1988). Ill-Posed problems in early vision. Proceedings of the IEEE, 76(8):869-889.

8. Calvetti D., Reichel L., Sgallari F., Spaletta G.(2000). A regularizing Lenclos iteration method for undetermined linear systems. Journal of Computation and Applied Mathematics, 115:101-120. 
9. Hansen P.C, O'Leary D.P. (1993). The use of the L-curve in the regularization of discrete ill posed problems. SIAM Journal on Scientific Computing, 14:1487-1503.

10. Wong H.S., Ling G. (2000). Application of evolutionary programming to adaptive regularization in image restoration. IEEE Transactions on Evolutionary Computation, 4(4):309-326.

11. Chan T.F., Wong C.K. (1998).Total variation blind deconvolution (1998).IEEE Transactions on Image Processing, 7(3):370-395.

12. Eberhart, R.C.,Kennedy, J. (1995). A new optimizer using particle swarm theory. Proceedings from the Sixth International Symposium on Micro Machine and Human Science, Nagoya, Japan, IEEE Service Center, Piscataway, NJ, (pp. 39-43).

13. Shi Y., Eberhart R.C .(1999). Empirical study of particle swarm optimization [A].Washington,DC: Proceedins of the 1999 congress on, Evolutionary Computation, 3:1927-1930

14. Liao H.Y., NG M.K. (2011). Blind deconvolution using generalized cross-validation approach to regularization parameter estimation. IEEE Trans. Image Processing, 20(3):670-680.

15.Chantas G.,Galatsanos N.,Molina R.,et al(2010). Variational Bayesian image restoration with a product of spatially weighted total variation image priors. IEEE Trans. Image Processing, $19(2): 351-362$.

16. LI W.H., LI Q., Gong W.G., et al (2012). Total variation blind deconvolution employing split Bregman iteration. ELSEVIER Journal of Visual Communication and Image Representation, 23(3):409-417. 